University of Nebraska - Lincoln

DigitalCommons@University of Nebraska - Lincoln

\title{
Similar traits, different genes? Examining convergent evolution in related weedy rice populations
}

Carrie S. Thurber

University of Massachusetts, cthurber@cns.umass.edu

Melissa H. Jia

USDA-ARS, melissa.jia@ars.usda.gov

Yulin Jia

USDA-ARS, yulin.jia@ars.usda.gov

Ana L. Caicedo

University of Massachusetts, caicedo@bio.umass.edu

Follow this and additional works at: https://digitalcommons.unl.edu/usdaarsfacpub

Thurber, Carrie S.; Jia, Melissa H.; Jia, Yulin; and Caicedo, Ana L., "Similar traits, different genes?

Examining convergent evolution in related weedy rice populations" (2012). Publications from USDA-ARS / UNL Faculty. 1127.

https://digitalcommons.unl.edu/usdaarsfacpub/1127

This Article is brought to you for free and open access by the U.S. Department of Agriculture: Agricultural Research Service, Lincoln, Nebraska at DigitalCommons@University of Nebraska - Lincoln. It has been accepted for inclusion in Publications from USDA-ARS / UNL Faculty by an authorized administrator of DigitalCommons@University of Nebraska - Lincoln. 


\title{
Similar traits, different genes? Examining convergent evolution in related weedy rice populations
}

\author{
CARRIE S. THURBER,$*$ MELISSA H. JIA $\uparrow \uparrow$ YULIN JIA $\uparrow$ and ANA L. CAICEDO* \\ *Biology Department, University of Massachusetts, Amherst, MA 01003, USA, †USDA-ARS Dale Bumpers National Rice \\ Research Center, Stuttgart, AR 72160, USA
}

\begin{abstract}
Convergent phenotypic evolution may or may not be associated with convergent genotypic evolution. Agricultural weeds have repeatedly been selected for weed-adaptive traits such as rapid growth, increased seed dispersal and dormancy, thus providing an ideal system for the study of convergent evolution. Here, we identify QTL underlying weedy traits and compare their genetic architecture to assess the potential for convergent genetic evolution in two distinct populations of weedy rice. $F_{2}$ offspring from crosses between an indica cultivar and two individuals from genetically differentiated U.S. weedy rice populations were used to map QTL for four quantitative (heading date, seed shattering, plant height and growth rate) and two qualitative traits. We identified QTL on nine of the twelve rice chromosomes, yet most QTL locations do not overlap between the two populations. Shared QTL among weed groups were only seen for heading date, a trait for which weedy groups have diverged from their cultivated ancestors and from each other. Sharing of some QTL with wild rice also suggests a possible role in weed evolution for genes under selection during domestication. The lack of overlapping QTL for the remaining traits suggests that, despite a close evolutionary relationship, weedy rice groups have adapted to the same agricultural environment through different genetic mechanisms.
\end{abstract}

Keywords: flowering time, Oryza sativa, Quantitative trait loci, red rice, shattering

Received 14 March 2012; revision received 18 October 2012; accepted 21 October 2012

\section{Introduction}

The repeatability of adaptive evolution is an outstanding question in biology. The presence of similar traits in independently evolved lineages has often been documented (e.g. Schluter et al. 2004), and it has recently become possible to determine the extent to which this is a result of similar changes in shared genetic systems (Stinchcombe \& Hoekstra 2008). Shared genetic biases among taxa that could result in disproportionate use of the same genes are often invoked to explain the occurrence of trait convergence (e.g. Hodin 2000; Schluter et al. 2004). These biases have been traditionally believed to be more likely among closely related species, suggesting that convergent phenotypic evolution among relatives is more likely attributable to shared

Correspondence: Ana L. Caicedo, Fax: 413 545-3243;

E-mail: caicedo@bio.umass.edu genetic mechanisms (e.g. Arendt \& Reznick 2007). To date, however, studies that identify underlying genes have revealed that convergent phenotypes can be due to shared or distinct genes, as well as shared or distinct mutations, both in closely and distantly related taxa (e. g. Yoon \& Baum 2004; Rompler et al. 2006; Steiner et al. 2008; Manceau et al. 2010; Powles \& Yu 2010; Remigereau et al. 2011; Smith \& Rausher 2011). Because patterns have been slow to emerge, the extent to and circumstances under which convergent phenotypic evolution is due to shared genetic mechanisms is currently an active area of inquiry.

Plants evolving in the agricultural environment offer many examples of convergent phenotypic evolution. For example, although domesticated in different world regions, many cultivated grasses have experienced similar selective pressures by humans; crop grasses have been selected for alterations in seed traits, annual life cycles, increased selfing and decreased seed shedding 
(Purugganan \& Fuller 2009). Similarly, trait convergence is often also evident in agricultural weeds - highly competitive plants that repeatedly invade the disturbed cropland soils (Basu et al. 2004). Despite sometimes being unrelated, agricultural weeds often converge on similar adaptive traits such as rapid growth, high seed production, increased seed dispersal and deep roots (Harlan \& DeWet 1965; Ellstrand et al. 2010). Little is currently known about the genetics underlying the evolution of these so-called 'weedy' traits, but the preponderance and diversity of agricultural weeds makes these ideal systems for studies of the genetic basis of convergent evolution.

Rice fields worldwide are often invaded by a weedy type of rice known as weedy or red rice (Oryza sativa L). Weedy rice is a major agricultural pest, as it is an aggressive competitor that spreads rapidly and drives down the quality of the rice harvest. Moreover, because it is closely related to the crop it invades, weedy rice is difficult to detect in its early growth stages and hard to control with herbicides (Vaughan et al. 2001). While limited, studies of weedy rice in various world regions suggest repeated origins of weedy populations (see Olsen et al. 2007). The presence of multiple populations of weedy rice around the world and their convergence on some typical weed-adaptive traits offer a unique opportunity to the study of convergent evolution at various geographic scales.

In the United States, where over 30\% rice fields are infested with weedy rice (Shivrain et al. 2009), two major morphologically and genetically differentiated populations of weedy rice co-occur. The Straw Hulled (SH) group most closely resembles cultivated rice with straw-coloured hulls and slightly larger grains; the Black Hulled \& Awned (BHA) group often resembles wild rice, with black- or brown-coloured hulls, small grains and long awns (Vaughan et al. 2001; Londo \& Schaal 2007; Reagon et al. 2010). Genome-wide assessments of polymorphism indicate that $\mathrm{SH}$ and BHA weedy populations are more closely related to indica and aus variety groups of domesticated Asian rice, respectively, than to other major cultivated or wild Oryzas (Reagon et al. 2010). Although there is debate over how many times Asian rice was domesticated, it is well accepted that cultivated rice was domesticated from Asian wild rice (Orzya rufipogon/Oryza nivara), with subsequent diversification of variety groups. Cultivated rice varieties are thus genetically differentiated, and the aus and indica putative ancestors of U.S. weedy rice groups are more closely related to each other (and more likely to share a domestication origin) than they are to the japonica cultivars grown in the United States (Garris et al. 2005; Caicedo et al. 2007). The origins of U.S. weedy rice from crop ancestors suggest that the evolution of weedy traits in these groups could be a process of 'de-domestication,' whereby selection favours reversions of domestication traits to forms characterizing wild species. This in turn suggests a different level at which convergent genetic evolution could occur: convergence of weedy and wild traits could be acquired through mutations in the same genes that were targeted during domestication.

U.S. weedy rice exhibits many traits that are associated with the persistence of weeds, such as increased seed dormancy and seed shattering, faster growth, taller plants and modified flowering times (Delouche et al. 2007; Shivrain et al. 2010). We have previously characterized some of these weedy traits relative to the putative cultivated progenitors and have noted different degrees of phenotypic convergence. For example, weedy rice from both $\mathrm{SH}$ and BHA are highly prone to shattering, a trait that is absent in the domesticated progenitors (Noldin et al. 1999; Thurber et al. 2010) (Table S1, Supporting information). Likewise, higher growth rates have been observed for $\mathrm{SH}$ and a subpopulation of BHA compared with their ancestors (Reagon et al. 2011). In contrast, flowering time (i.e. heading date) is strongly in differentiated in both weed groups compared with their cultivated progenitors, but the shifts are in opposite directions: under day neutral conditions (12 hours light), SH flowers significantly earlier than indica, whereas BHA groups flower significantly later than aus (Reagon et al. 2011) (Table S1, Supporting information). In field conditions, blackhull weeds also typically flower later than strawhull weeds (Shivrain et al. 2009). Thus, although the same trait has been affected in the course of weed evolution, there has not been convergence on a single phenotypic value. A similar situation is seen for plant height. Weedy rice shows a range of heights, but under-controlled conditions, $\mathrm{SH}$ weeds are generally shorter than their indica progenitors, and BHA weeds are generally taller than the aus (Reagon et al. 2011) (Table S1, Supporting information). In the field, both weed groups tend to be taller than the local japonica crop (Shivrain et al. 2009), likely driven by the recent selection for semi-dwarf highyielding rice plants (see Asano et al. 2007). Remarkably, these divergent weedy phenotypes have evolved under near identical selective pressures, as weedy rice from both populations is often found in the same rice fields (Shivrain et al. 2010).

Given the convergence of phenotypic values for some traits and divergent evolution for others, we are interested in determining to what extent common loci underlie weedy trait evolution in U.S. weedy rice groups. In particular, we would like to answer the following questions: (i) Do weedy rice populations share QTL underlying convergent traits? (ii) Do weedy rice populations utilize different QTL for divergent phenotypes? 
(iii) Are QTL shared when weedy traits mimic those of wild rice? We hypothesized that, given the weeds' descent from cultivated ancestors sharing a domestication origin, U.S. weedy rice groups are likely to have shared biases leading to use of the same genes in weedy trait evolution. We conducted crosses of each US weed with a putative progenitor or close relative to capture the genetic differences that have accumulated since each weed group diverged from a cultivated background. Using $\mathrm{F}_{2}$ populations, we carried out QTL mapping of four quantitative traits that have either converged or diverged between weedy rice groups, as well as two qualitative traits specific to the BHA group that have converged on wild rice phenotypes. Our goal was not to identify causal genes but to begin assessment of the degree to which shared genomic regions underlie weedy traits. We find that, in most cases, similar genomic regions are not involved in traits characterizing weedy rice groups; the exception to this is flowering time, which, although divergent among groups, may involve modification of alternative alleles at loci involved in the Oryza flowering time pathway.

\section{Methods}

\section{Plant materials}

We created two mapping populations ( $\mathrm{S}$ and $\mathrm{B}$ ) by crossing two weedy rice individuals (SH-RR09 and BHA-RR20) with a single indica cultivar Dee Geo Woo Gen (DGWG), which in both cases was the pollen donor. The weedy rice parents are representatives of the SH and BHA populations of U.S. weedy rice as determined by population structure assessments (Reagon et al. 2010). The indica cultivar group was chosen as a parent because this group is putatively ancestral to the $\mathrm{SH}$ weed group and is closely related to the BHA ancestor, aus (Caicedo \& Purugganan 2005; Garris et al. 2005; Reagon et al. 2010); multiple attempts to crossBHA weeds with aus cultivars failed. Weed and crop parents were selected to maximize phenotypic differences in potential weed-adaptive traits based on previous growth chamber data (Table 1). Reciprocal crosses did not work for the BHA $\times$ indica cross, thus assessment of maternal effects was not attempted. All parents are inbred lines, self-fertilized multiple times prior to acquisition from the International Rice Research Center and the USDA, and further selfed 1-3 generations in our laboratory prior to crossing. The resulting $F_{1}$ plants largely showed phenotypes intermediate between the two parents; the single exception was the B population $\mathrm{F}_{1}$, which produced seeds with black hulls and awns suggesting that these traits are controlled by few genes in which the BHA allele is dominant. $F_{1}$ plants were confirmed to be the result of crosses and were allowed to self to create the $F_{2}$ seeds used for mapping.

Approximately $250 \mathrm{~F}_{2}$ seeds per population, offspring from a single $F_{1}$ for each cross, were sown in a greenhouse in Amherst, MA on 1 April 2010 in four-inch pots set in two-inch trays of ten pots each. Seeds were heat treated for twelve hours at $37^{\circ} \mathrm{C}$, and the hulls were partially or totally removed prior to planting to eliminate dormancy. Approximately 25 trays per population were interspersed randomly throughout the greenhouse. Three replicate pots of each parental line were also sown in a single tray in the greenhouse to serve as phenotypic controls. Water was maintained at a height of approximately one to two inches in trays to keep soil moist, and fertilizer (NPK 15-16-17) was applied weekly with an added iron application on alternate weeks. Environmental conditions varied seasonally, with day-length varying between approximately 10 and $15 \mathrm{~h}$ light and temperature varying between 20 and $27^{\circ} \mathrm{C}$. The emergence date of $\mathrm{F}_{2}$ seedlings was not uniform within each population despite dormancy-releasing treatment. Due to inadequate $F_{2}$ germination, two additional waves of planting were performed with approximately 100 seeds on 22 April 2010 and 13 May 2010. As different planting dates likely put seedlings under different light and temperature environments, we compared trait distributions and averages for heading date, plant height and seed shattering across planting waves. No differences were observed for any trait (data not shown). Additionally, no differences were observed in the QTL detected using only the first wave individuals and the full data set; thus, we used all three waves to increase our statistical power. Final experimental plants were harvested on 20 January 2011. $184 \mathrm{~S}$ population and 159 B population individuals were phenotyped and genotyped successfully and were usable for mapping.

\section{Trait evaluation}

Four quantitative traits, heading date, plant height, growth rate and seed shattering, were evaluated in each $\mathrm{F}_{2}$ population (Tables S2 and S3, Supporting information). Additionally, two qualitative traits, hull colour and awns, were evaluated in the B population, as these traits did not differ between $S$ population parents (Table S3, Supporting information). Heading date was measured in days from the date of seedling emergence until the first panicle had emerged halfway from the boot. Panicles were bagged at this stage to ensure selfing and prevent loss of seeds. Plant height was measured in centimetres, at heading, from the base of the plant at the soil to the tip of the tallest panicle excluding awns. Growth rate was calculated by dividing plant 
Table 1 Phenotypes of the parental lines crossed to create $F_{2}$ mapping populations. Plants were initially chosen for their different phenotypes in the growth chamber. Greenhouse measurements are averages of three plants. Growth chamber measurements are averages of two plants, and have been reported in Thurber et al. (2010) and Reagon et al. (2011). Numbers in parentheses are standard deviations

\begin{tabular}{|c|c|c|c|c|c|c|}
\hline Parental line & Plant height $(\mathrm{cm})$ & Heading date (days) & Growth rate (cm/day) & Seed shattering* $(\mathrm{g})$ & Hull color & Awn \\
\hline \multicolumn{7}{|l|}{ Greenhouse } \\
\hline Weedy-SH & $76.8(5.12)$ & $107.3(0.96)$ & $0.716(0.04)$ & $0(0)$ & 0 & 0 \\
\hline Cultivated & $42.7(7.02)$ & $101(3.46)$ & $0.423(0.06)$ & $28.4(7.99)$ & 0 & 0 \\
\hline Weedy-BHA & $104.7(3.79)$ & $116(1.73)$ & $0.903(0.04)$ & $5.5(1.84)$ & 1 & 1 \\
\hline \multicolumn{7}{|c|}{ Growth Chamber } \\
\hline Weedy-SH & $83(3.54)$ & $60(4.95)$ & $1.383(0.04)$ & $0.3(0.06)$ & 0 & 0 \\
\hline Cultivated & $59(9.19)$ & 109 (23.33) & $0.541(0.19)$ & $60.9(23.02)$ & 0 & 0 \\
\hline Weedy-BHA & $67(33.23)$ & $116(13.43)$ & $0.578(0.33)$ & $7.2(3.08)$ & 1 & 1 \\
\hline
\end{tabular}

*Seed shattering was measured as described in Thurber et al. 2010 using the Breaking Tensile Strength method.

height by heading date to get an average rate in $\mathrm{cm} /$ day. Seed shattering was measured in grams of force required to remove the seed from the panicle; measurements were taken from 10 mature seeds collected 30 days after heading and averaged per individual as described in Thurber et al. (2010). Hull colour was scored as straw (0) and black (1) on seeds collected thirty days after heading. A small number $(<20)$ of individuals showed brown or gold hull colours and were not considered for analysis. Awns were recorded as presence (1) vs. absence (0) at the same time hull colour was scored. Pairwise Pearson's correlation coefficients were calculated in Excel for trait values in each mapping population.

Broad-sense heritability $\left(H^{2}\right)$ for each trait in each population was calculated as in $\mathrm{Xu}$ et al. (2009). Briefly, variance for each parental line was estimated in the greenhouse environment, and the average of the parental variances was used as the environmental variance $\left(V_{\mathrm{e}}\right)$ for each mapping population. $V_{\mathrm{e}}$ was subtracted from the total phenotypic variance of the $F_{2}$ population $\left(V_{\mathrm{p}}\right)$ to obtain the genetic variance $\left(V_{\mathrm{g}}\right)$. Because estimates from our parental lines likely underestimate $V_{\mathrm{e}}$, a maximum $H^{2}$ was calculated as $V_{\mathrm{g}} / V_{\mathrm{p}}$ for each trait.

\section{Marker analysis}

DNA was extracted from frozen tissue collected from greenhouse grown $\mathrm{F}_{2}$ plants using a CTAB method (Reagon et al. 2010). Over 188 microsatellite (SSR) markers from previously published studies (e.g. Chen et al. 1997; McCouch et al. 2002) were genotyped in the three parental lines. SSR markers are identified as numbers that correspond to the 'RM' markers from previous studies. Additionally, two and six insertion-deletion (indel) markers were adapted from Shen et al. (2004) for the $S$ and B populations, respectively. These were given names R\#M\#. Lastly, five additional SNP markers (labelled as c\#p\# corresponding to the chromosome and physical position of the marker) and three additional indel markers (labelled i\#) were developed from wholegenome sequence data (K. E. Hyma \& A. L. Caicedo, unpublished data).

Indel and SSR markers were PCR amplified similar to Panaud et al. (1996) except that the reaction volume was reduced to $15 \mu \mathrm{l}$ and PCR cycling conditions were as follows: $94^{\circ} \mathrm{C}$ for $5 \mathrm{~min}$, followed by 35 cycles of $94^{\circ} \mathrm{C}$ for $30 \mathrm{~s}, 55^{\circ} \mathrm{C}$ for $30 \mathrm{~s}$, and $68^{\circ} \mathrm{C}$ for $1 \mathrm{~min}$; finished by $5 \mathrm{~min}$ at $72^{\circ} \mathrm{C}$. SNP markers were amplified as above in two separate reactions (one with a forward primer unique to the weedy allele and one unique to the cultivated allele), with the exception that a TouchDown PCR was carried out starting 10 degrees above the expected melting temperature $\left(T_{\mathrm{m}}\right)$ and decreasing $1^{\circ}$ every cycle, followed by 25 cycles at the target $T_{\mathrm{m}}$. Indel and SNP marker genotypes were directly scored from 2\% agarose gels. Amplified SSR products were run on an ABI 3130XL genetic analyzer at the Genomics Resource Laboratory at the University of Massachusetts. FSA files were analysed using the PeakScanner software to determine the sizes of bands. All marker genotypes were scored as 0,1 or 2 depending on whether the individual was homozygous for the cultivated allele, heterozygous or homozygous for the weedy allele, respectively.

Marker segregation analysis was carried out using chi-square tests to detect significant distortion from Mendelian inheritance. Linkage maps were created using the Kosambi map function under default conditions in $\mathrm{R} / \mathrm{qtl}$, resulting in maps of approximately 1550 -1700 cM. A ripple function was used to test alternative marker order. Only three rearrangements were suggested, and subsequent mapping did not lead to dif- 
ferences in QTL detected, positions or LOD scores; we thus kept the original marker order, which is consistent physical marker location and previous rice maps. The average interval size of our maps is approximately $29.8 \mathrm{cM}$ for the S population and $24.1 \mathrm{cM}$ for the B population, with a minimum of $1.4 \mathrm{cM}$ and a maximum of $91.5 \mathrm{cM}$. Although some large gaps occur, marker positions were found to be in similar locations as previously published maps and should be sufficient for detecting most large-effect loci (e.g. Thomson et al. 2003; Lee et al. 2005).

\section{QTL mapping}

The normality of phenotypic data was checked using Normal Quantile Plots (Tan et al. 2004). Non-normal data were $\log$ transformed. If transformation was unsuccessful, nonparametric analysis was performed (Tilquin et al. 2001). For normalized traits, QTL were identified first using single-marker analysis (SMA) as a rough scan of the individual markers, and then using Composite Interval Mapping (CIM) to get more accurate positions and significance values. Both analyses were run in WinQTL Cartographer (Wang et al. 2012). SMA was run under default conditions; CIM was run using forward-backward regression (Model 6; probability of inclusion/exclusion of marker cofactors of 0.1 ; window size $10 \mathrm{cM}$ ) and a walk speed of $8 \mathrm{cM}$. Nonparametric analysis was performed on seed shattering using a Kruskal-Wallis rank sum test (K-W test) in $\mathrm{R} / \mathrm{qtl}$. Mapping of the hull colour and awns traits was performed in $\mathrm{R} / \mathrm{qtl}$ using the binary mode under default conditions. LOD thresholds for normalized traits were assessed via permutations with 1000 replicates, with most values being close to 3.5 (Table 2). To make sure as many biologically significant QTL as possible were detected given the variety of mapping analyses performed and the low marker density, we report all QTL with LOD scores over 2.0. The locations of the QTL identified in this study were compared to previously published QTL using the 'QTL' search feature on Gramene (http://www.gramene.org/).

\section{Results}

\section{Phenotypes}

To see whether environment had an effect on trait differentiation, we compared phenotypes of the parents under greenhouse conditions with phenotypes previously obtained in the growth chamber (Table 1). Growth chamber phenotypes were originally used to select mapping parents, and the greenhouse environment differed in having more variable temperatures and seasonally variable day-length. Phenotypic differences among environments were seen for some traits. Most strikingly, the weedy-SH parent had an increase in over 47 days in heading date under the greenhouse environment. This is consistent with the short day flowering behaviour seen in many rice varieties (Yano et al. 2000), given the differences in day-length between the growth chamber (12 h) and greenhouse (day-length often exceeds $12 \mathrm{~h}$ in the summer). In contrast, heading date of the weedy-BHA and the cultivated parent were consistent across environments, suggesting limited photoperiod sensitivity. The cultivated parent showed nearly half as much seed shattering resistance in the greenhouse, while there was no sizable change in the weeds' shattering abilities. Plant height nearly doubled for the weedy-BHA parent in the greenhouse, while the other two parents remained close to growth chamber values. Growth rate values changed for both weed parents in the greenhouse, but consistently exceeded the crop parent. Despite environmental influence, phenotypic differences between weed and crop parents were still evident for most traits under greenhouse conditions (Table 1).

We examined the phenotypic distributions of all traits in the $F_{2}$ populations. For qualitative traits, segregation of hull colour fits the 3:1 ratio expected for a trait controlled by a single gene, while for awns, there is an excess of weedy parent phenotypes suggesting the involvement of more than one locus $\left(\chi^{2}=9.358\right.$, $P<0.01$ ) (Table 3; Fig. 1B). For quantitative traits, continuous, nearly normal distributions were observed for plant height, heading date and growth rate, and normality could not be rejected for the $\log _{10}$ values of these traits in either population $(\alpha=0.01)$. In contrast, normality was rejected for seed shattering in both populations and attempts to normalize this trait failed. Transgressive segregation was seen in all traits in both populations yet was most noticeable for heading date (Fig. 1A). Trait means were fairly similar between the two populations for all quantitative traits. The distributions of plant height and growth rate were nearly identical between populations, suggesting similar genetic architectures. For both heading date and seed shattering, multiple peaks were observed in the $S$ population, a distribution type often attributed to a locus of major effect and a few of minor effect, while the B population distributions suggested multiple weaker-effect loci (Fig. 1A).

A weak but significant positive correlation was found only between heading date and plant height in both populations $(r=0.189-0.204) \quad$ (Table S4, Supporting information). Stronger positive correlations between these traits $(r=0.467-0.76)$ have been reported in other 
Table 2 QTL for quantitative traits detected in the $\mathrm{F}_{2}$ populations

\begin{tabular}{|c|c|c|c|c|c|c|c|c|c|c|}
\hline \multirow[b]{2}{*}{ QTL* } & \multirow[b]{2}{*}{ Chr } & \multirow[b]{2}{*}{ Position (cM) } & \multirow[b]{2}{*}{ Nearest marker } & \multicolumn{2}{|c|}{ LOD score $\dagger$} & \multirow[b]{2}{*}{$\mathrm{R}^{2} \boldsymbol{q}$} & \multicolumn{3}{|c|}{ Phenotypic means } & \multirow[b]{2}{*}{ Increased effect** } \\
\hline & & & & CIM§ & NP & & 0 & 1 & 2 & \\
\hline \multicolumn{11}{|c|}{ Seed shattering } \\
\hline qSS1b & 1 & 189 & 104 & $\mathrm{~N} / \mathrm{A}$ & 2.23 & $\mathrm{~N} / \mathrm{A}$ & 19.55 & 14.21 & 13.02 & Cultivated \\
\hline qSS2s & 2 & 15.2 & 236 & $\mathrm{~N} / \mathrm{A}$ & 6.85 & $\mathrm{~N} / \mathrm{A}$ & 13.14 & 8.12 & 4.44 & Cultivated \\
\hline qSS11s & 11 & 0.1 & 332 & $\mathrm{~N} / \mathrm{A}$ & 2.71 & $\mathrm{~N} / \mathrm{A}$ & 9.16 & 10.21 & 3.84 & Cultivated \\
\hline qSS12s & 12 & 21.8 & 3246 & $\mathrm{~N} / \mathrm{A}$ & 2.43 & $\mathrm{~N} / \mathrm{A}$ & 7.06 & 7.05 & 12.7 & Weedy-SH \\
\hline \multicolumn{11}{|c|}{ Heading date } \\
\hline qHD4b & 4 & 45.2 & R4M17 & 2.45 & $\mathrm{~N} / \mathrm{A}$ & 16.8 & 118.7 & 118.95 & 110.72 & Cultivated \\
\hline qHD $8.1 b$ & 8 & 17.8 & 25 & 2.78 & $\mathrm{~N} / \mathrm{A}$ & 10.3 & 112.05 & 114.83 & 123.4 & Weedy-BHA \\
\hline qHD8.2b & 8 & 78.6 & 44 & 2.59 & $\mathrm{~N} / \mathrm{A}$ & 16.6 & 119.2 & 111.96 & 120.94 & Weedy-BHA \\
\hline qHD4s & 4 & 112.7 & 451 & 2.08 & $\mathrm{~N} / \mathrm{A}$ & 4.4 & 114.82 & 122.3 & 126.82 & Weedy-SH \\
\hline qHD7s & 7 & 24.3 & RID12 & 2.71 & $\mathrm{~N} / \mathrm{A}$ & 5.3 & 123.85 & 129.26 & 109.17 & Cultivated \\
\hline qHD $8.1 \mathrm{~s}$ & 8 & 20.9 & 310 & 18.9 & $\mathrm{~N} / \mathrm{A}$ & 32 & 96.55 & 125.94 & 139.93 & Weedy-SH \\
\hline qHD8.2s & 8 & 120.5 & 284 & 2.56 & $\mathrm{~N} / \mathrm{A}$ & 38.6 & 111.78 & 121.55 & 132.11 & Weedy-SH \\
\hline \multicolumn{11}{|l|}{ Plant height } \\
\hline qPH1b & 1 & 162.9 & 5407 & 6.43 & $\mathrm{~N} / \mathrm{A}$ & 22.7 & 56.72 & 66.8 & 68.95 & Weedy-BHA \\
\hline qPH5b & 5 & 112.1 & 146 & 3.33 & $\mathrm{~N} / \mathrm{A}$ & 13.7 & 57.8 & 64.9 & 68.4 & Weedy-BHA \\
\hline qPH4s & 4 & 31.2 & 417 & 2.45 & $\mathrm{~N} / \mathrm{A}$ & 5.5 & 66.5 & 63.14 & 78.79 & Weedy-SH \\
\hline qPH8s & 8 & 193.5 & 477 & 2.53 & $\mathrm{~N} / \mathrm{A}$ & 14.3 & 68.55 & 61.37 & 75.05 & Weedy-SH \\
\hline qPH10s & 10 & 0.1 & 239 & 2.05 & $\mathrm{~N} / \mathrm{A}$ & 18.6 & 71.74 & 65.83 & 63.47 & Cultivated \\
\hline
\end{tabular}

*' $\mathrm{b}$ ' and ' $\mathrm{s}$ ' designations in the QTL identifier refer to the B and S mapping populations, respectively.

${ }^{\dagger}$ LOD thresholds determined by permutation tests for each trait were: $S$ population heading date 8.3 , plant height 3.5 , growth rate 3.5; B population heading date 3.5, plant height 3.6, growth rate 3.5. All QTL with LOD above 2.0 are reported in the table.

"Phenotypic means calculated for the cultivated homozygote (0), heterozygote (1) and weedy homozygote (2).

${ }^{\S}$ Bold values are also $>2.0$ under single-marker analysis.

$" R^{2}$ indicates the percentage of phenotypic variation explained by the putative QTL.

**Increased effect is the source of the allele causing an increase in the phenotypic value.

Table 3 Segregation and mapping of qualitative trait loci. In both cases, the weedy phenotype (awns, black hull) is dominant as these were seen in the F1 generation

\begin{tabular}{|c|c|c|c|c|c|c|c|}
\hline \multirow[b]{2}{*}{ Trait } & \multicolumn{3}{|c|}{ Phenotypic ratio } & \multirow[b]{2}{*}{ Chr. } & \multirow[b]{2}{*}{ Linked markers } & \multirow[b]{2}{*}{ Distance $(\mathrm{cM})$} & \multirow[b]{2}{*}{ LOD } \\
\hline & Dominant & Recessive & $\chi^{2}$ & & & & \\
\hline \multirow[t]{3}{*}{ Awns } & 136 & 23 & 9.36 & 3 & c3p24 & 114.6 & 2.14 \\
\hline & & & & 9 & 107 & 89.5 & 2.01 \\
\hline & & & & 11 & 202 & 37.8 & 2.51 \\
\hline \multirow[t]{2}{*}{ Hull colour } & 94 & 40 & 0.236 & 1 & 9 & 75.2 & 2.83 \\
\hline & & & & 4 & 6748 & 127.2 & 2.81 \\
\hline
\end{tabular}

studies of greenhouse and field grown cultivated rice (see Bres-Patry et al. 2001; Lee et al. 2005).

A maximum broad-sense heritability value was calculated for all quantitative traits in each population (Table S5, Supporting information). Despite the evidence for environmental effects on some of these traits in parents, heritabilities in our greenhouse environment were fairly high, ranging from approximately $67 \%$ for seed shattering to nearly $100 \%$ for heading date. Although our heritability values are likely to be overestimates, particularly in the case of plant height (Table S5, Supporting information), heritabilities were also remarkably similar for each trait across mapping populations.

\section{Marker linkage map}

Of the 188 SSR tested, 31\% were polymorphic between the cultivar and weedy-SH parent and $45 \%$ were 

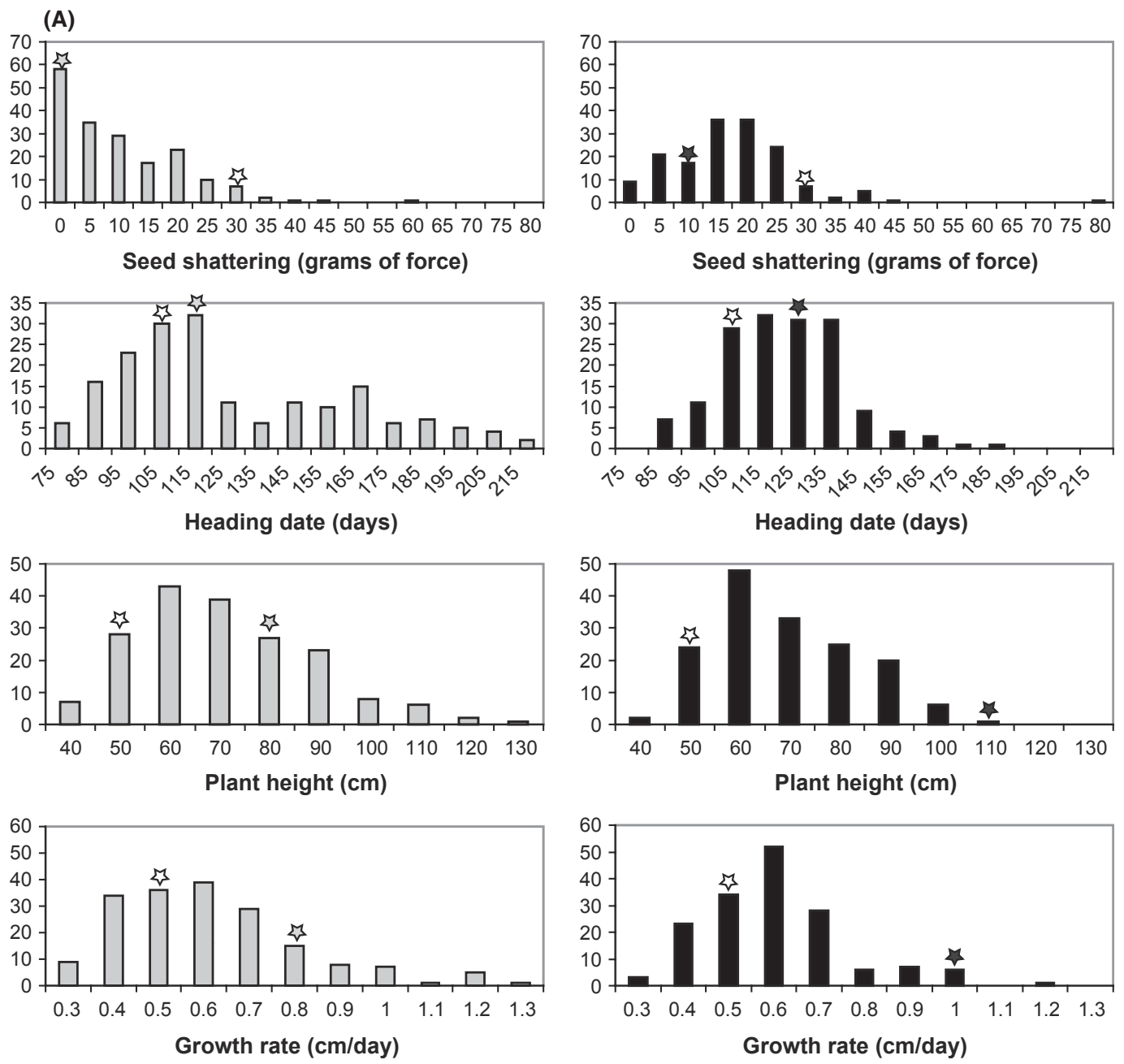

(B)
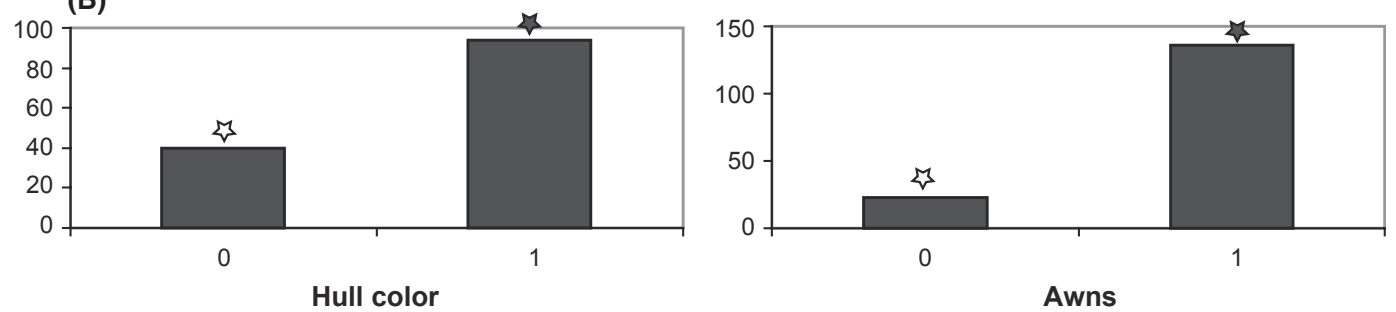

Fig. 1 Frequency distributions of traits in the $F_{2}$ populations. Grey bars represent traits measured in the $S$ population, while black bars represent traits measured in the B population. White stars correspond to trait values for the cultivated parent and filled stars to the respective weed parent. The vertical axis of each figure represents the number of individuals. (A) Phenotypic distributions for quantitative traits. (B) Phenotypic distributions for qualitative traits in the B population.

polymorphic between the cultivar and weedy-BHA. These low levels of polymorphism are not unusual; population structure analyses cannot differentiate $\mathrm{SH}$ weeds from indica cultivars, and BHA weeds share alleles with both aus and indica (Reagon et al. 2010), making finding segregating markers among parents difficult. As expected, given putative ancestry, less polymorphism was seen between the $\mathrm{SH}$ weed and indica parent. A total of 54 and 72 SSR markers were usable for the $\mathrm{S}$ and $\mathrm{B}$ populations, respectively. With the addition of polymorphic indels and SNPs, we mapped using 63 markers for the $\mathrm{S}$ population and 82 markers for the $\mathrm{B}$ population (Tables S6 and S7, Supporting information).

Segregation distortion at the $P<0.01$ level was seen in 14 and 12 markers in the $\mathrm{S}$ and $\mathrm{B}$ populations, respectively. About half of the distorted markers had excess weedy alleles, while the rest had excess cultivated alleles. Only two markers were distorted in both 
populations. The presence of QTL linked to distorted markers can affect detection; yet, distorted markers do not cause false-positive associations and are not a problem if randomly distributed across a genetic map (Zhang et al. 2010). When distorted markers were removed, we did not detect additional QTL for any trait; loss of a hull colour QTL (chromosome 1) was observed, as it is linked to a distorted marker (9), and one QTL for heading date (chromosome 4) in the $\mathrm{S}$ population dropped below the LOD 2.0 threshold.

\section{Mapping quantitative traits}

Seed shattering. Three shattering QTL were identified in the S population (Fig. 2; Table 2; Fig. S1, Supporting information). One is located on chromosome 2 near position $15.2 \mathrm{cM}$ (qSS2s), another is located on chromosome 11 near position $0.1 \mathrm{cM}$ (qSS11s), and the third is located on chromosome 12 near position 21.8 (qSS12s). Weedy alleles at two QTL increase seed shattering ability as expected, given the weedy parent's propensity for shattering. Our QTL on chromosome 11 may be close or overlapping with one found in a cross between wild O. rufipogon and an indica cultivar, where the wild allele increased seed shattering (Cai \& Morishima 2000). In the B population, one QTL was identified on chromosome 1 near position $189 \mathrm{cM}$ (qSS1b) (Fig. 2; Table 2; Fig. S1, Supporting information). Weedy alleles at this QTL also work to increase seed shattering. This QTL is linked to a shared marker with a shattering QTL from a cross between O. rufipogon and a tropical japonica cultivar, where the wild allele increased seed shattering (Thomson et al. 2003). Additionally, a peak under the LOD threshold in the B population corresponding to a similar location as the $\mathrm{S}$ chromosome 11 QTL may indicate sharing of a minor-effect QTL we did not have the power to detect (Fig. S1, Supporting information).

Heading date. For the $\mathrm{S}$ population, four heading date QTL were identified with LOD > 2.0 (Fig. 2; Table 2; Fig. S1, Supporting information). One is located on chromosome 4 (qHD4s, $112.7 \mathrm{cM}$ ), another on chromo-
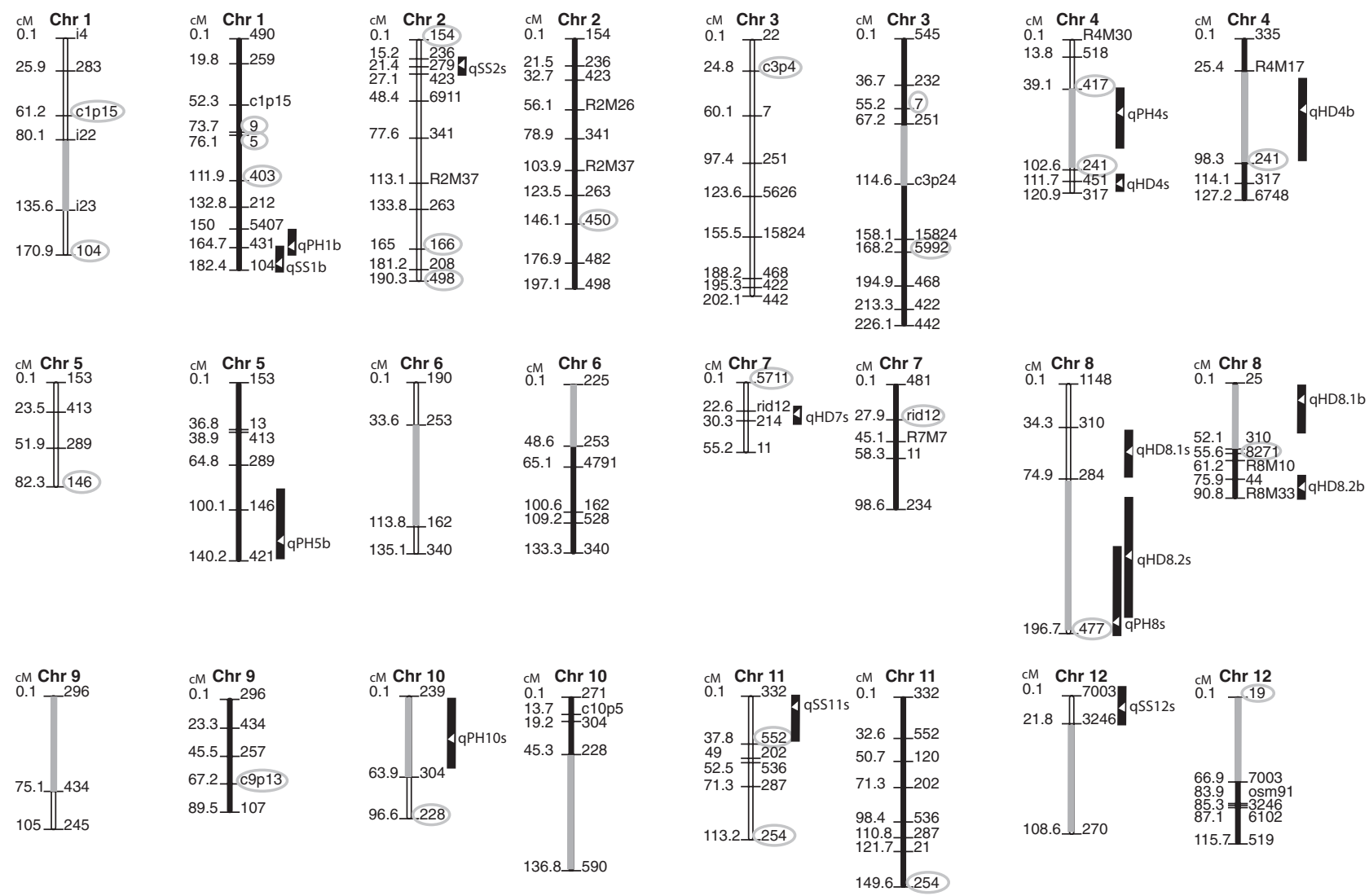

Fig. 2 Molecular linkage maps with positions of QTL for quantitative traits in both the S (left) and B populations (right). Markers with segregation distortion $(P<0.01)$ are circled in light grey. Black bars represent 1.5 LOD confidence intervals around QTL peaks, which are labelled with a white triangle. Grey bars represent gaps over $45 \mathrm{cM}$. Marker names are on the right side of the chromosome; marker positions, drawn to scale, are on the left. 
some 7 (qHD7s, $24.3 \mathrm{cM}$ ) and two on chromosome 8 (qHD8.1s, $20.9 \mathrm{cM}$; qHD8.2s, $120.5 \mathrm{cM}$ ). Weedy alleles at three of the QTL increase days to heading, consistent with the later flowering seen in the weedy parent in the greenhouse. The $\mathrm{S}$ population permutation LOD threshold was surprisingly high compared with other traits (Table 2); this may be a spurious effect of the bimodal phenotype distribution, which persisted somewhat after normalization of the trait (Manichaikul et al. 2007).

In the $\mathrm{B}$ population, three QTL were identified on chromosomes 4 (qHD4b, $45.2 \mathrm{cM}$ ) and 8 (qHD8.1b, $17.8 \mathrm{cM}$; qHD8.2b, $78.6 \mathrm{cM}$ ) (Fig. 2; Table 2; Fig. S1, Supporting information). Weedy alleles at two of the QTL increase heading date, consistent with the weedy parent's phenotype. It is possible that qHD8.1s and qHD8.1b share similar or linked causal genes as their peaks overlap with the corresponding QTL's 1.5 LOD interval and both include marker 310. Additionally, these QTL may be in a similar location as one identified by Yano et al. (1997) and Xiao et al. (1998), in mapping populations involving japonica by indica crosses (with the japonica allele reducing flowering time), and O. rufipogon by cultivated crosses (with wild alleles increasing flowering time). Recently, a candidate gene in this region was cloned (Wei et al. 2010; Cai et al. 2012; Yan et al. 2011). Additionally, qHD8.2s and qHD8.2b also seem localized to similar genomic areas; however, lack of shared markers in this region prevent us from determining whether these QTL overlap.

Plant height. Three QTL were detected for plant height in the S population (Fig. 2; Table 2; Fig. S1, Supporting information). Of these, qPH10s (chromosome 10, $0.1 \mathrm{cM})$ explains most of the variation followed by qPH8s (chromosome 8, $193.5 \mathrm{cM}$ ) and qPH4s (chromosome $4,31.2 \mathrm{cM})$. For $\mathrm{qPH} 4 \mathrm{~s}$ and $\mathrm{qPH} 8 \mathrm{~s}$, the weedy allele increases plant height, as expected, while for qPH10s the cultivated allele increases plant height. Although we do not share any neighbouring or linked markers with Li et al.'s (2006a) map from an indica and wild $O$. nivara cross, the markers associated with our chromosome 4 QTL are in similar physical locations. We do share a neighbouring marker with a QTL on chromosome 8 from Thomson et al. (2003) and with a QTL on chromosome 10 with Septiningsih et al. (2003), from populations involving crosses between japonica or indica cultivars with wild O. rufipogon; in all three studies, the wild allele increases plant height.

Two QTL were identified in the B population; one near position $162.9 \mathrm{cM}$ on chromosome 1 (qPH1b) and the other near position $112.1 \mathrm{cM}$ on chromosome 5 (qPG5b) (Fig. 2; Table 2; Fig. S1, Supporting information). The weedy allele at both of these QTL increases plant height, as expected. The peak marker for the QTL on chromo- some 1 (5407) is physically located at approximately 39.5 megabase pairs (mbp), which is very close to $S D 1$, a cloned gene of major effect that encodes a critical enzyme involved in gibberellin biosynthesis (Monna et al. 2002).

Growth rate. No significant growth rate QTL were detected in either population. Interestingly, despite clear importance of this trait for plant fitness and competiveness, we have found only one study mapping growth rate in Oryza (Li et al. 2006c), which may be indicative of its complex genetic basis. Although $\mathrm{Li}$ et al. found several QTL underlying growth rate, their measurements were based on dry weight accumulated over time and are likely not comparable to ours.

\section{Mapping qualitative traits}

Two significant loci were detected for hull colour, one on chromosome 1 near position $75.2 \mathrm{cM}$ and another on chromosome 4 near position $127.2 \mathrm{cM}$ (Table 3; Fig. S2, Supporting information). The locus on chromosome 1 may be novel. A locus on chromosome 4 controlling black to straw hull colour change, identified in a cross between an indica cultivar and O. rufipogon was cloned recently (Os04g0460000; approximately $22.78 \mathrm{mbp}$ ). Known as Bh4, this locus is physically close to our significant marker on chromosome 4 (approximately $29.7 \mathrm{mbp}$ ) (Zhu et al. 2011).

Three significant loci were detected for awn presence on chromosomes $3(114.6 \mathrm{cM}), 9(89.5 \mathrm{cM})$ and 11 (37.8 cM) (Table 3; Fig. S2, Supporting information). A QTL for awn length identified in a cross between an indica cultivar and wild O. rufipogon by Cai \& Morishima (2002) on chromosome 11 is close to our locus, yet we do not share any co-localized markers.

\section{Discussion}

The repeatability of evolution can be seen as convergent changes at the phenotypic and/or genetic level between organisms evolving under similar environmental conditions. Questions remain about the extent to which shared genes are likely to underlie trait convergence among distant and closely related organisms (Arendt \& Reznick 2007). Various species of weedy plants repeatedly invade agricultural fields and are often subjected to similar selective pressures, leading to convergent phenotypic evolution of putative weed-adaptive traits (Harlan \& DeWet 1965; Ellstrand et al. 2010). Because weedy red rice in the United States consists of two independently evolved groups descended from closely related cultivated ancestors (Reagon et al. 2010), we sought to determine whether similar genetic changes were involved in the evolution of weediness in these 
groups, and whether weedy traits could be attributed to variation present in wild and/or cultivated rice.

\section{Lack of convergent genetic evolution for convergent seed shattering in U.S. weedy rice}

Of all the traits that differentiate weedy rice from its cultivated progenitors, seed shattering is the one that most characterizes the weedy phenotype. Selection against shattering to facilitate harvesting is a hallmark of cereal domestication (Purugganan \& Fuller 2009). In contrast, efficient seed dispersal is likely crucial to weed fitness, allowing weeds to spread and increase their presence in the seed bank (Harlan \& DeWet 1965). We have previously shown that, despite separate origins, both U.S. weedy rice groups are highly shattering compared to their putative cultivated progenitors (Thurber et al. 2010). Thus, seed shattering is a trait for which true phenotypic convergence has occurred.

The genetic basis of loss of shattering in cultivated rice has been much explored, and sh4, a gene coding for a transcription factor involved in degradation of the abscission layer, has been identified as the most significant shattering gene selected on during domestication (Li et al. 2006b; Lin et al. 2007; Zhang et al. 2009). Studies have shown that all rice cultivars share a singlenucleotide substitution in sh4, which leads to loss of shattering (Zhang et al. 2009; Thurber et al. 2010). Recently, we found that both U.S. weedy rice groups possess the same 'non-shattering' substitution as cultivated rice (Thurber et al. 2010). This implies that weedy groups have re-acquired the shattering trait through involvement of other loci (see also $\mathrm{Zhu}$ et al. 2012). We have additionally shown that both weed groups have convergence of shattering at the morphological level formation and degradation of the abscission layer is similar among weedy groups, but distinct from shattering wild rice (Thurber et al. 2011). This phenotypic evidence suggests that similar genetic changes underlie convergence of shattering in weedy rice groups.

Surprisingly, both the phenotypic distributions in our mapping populations and identified QTL do not support convergent genetic changes in seed shattering between weeds. In the S population, a highly nonnormal distribution with multiple peaks suggests that a few major-effect genes contribute to this trait. In contrast, the more normal shattering distribution in the $\mathrm{B}$ population suggests involvement of multiple weakereffect loci. We identified three QTL for seed shattering in the $S$ population and one in the $B$ population, but these are located on four different chromosomes and do not appear shared among populations.

Mapping for shattering had to be carried out with a nonparametric approach, which increases the possibility of false negatives for loci of small effect. This likely affected our ability to detect QTL particularly in the B population, for which shattering seems to be a more polygenic trait. However, given that a QTL of large effect was detected for the $S$ population on chromosome 2 - one of the best characterized in both populations and given that shared markers on chromosome 1 did not lead to detection of the single B QTL in the S population, the difference in genetic architecture among weed individuals seems real. Thus, despite the potential of shared genetic biases due to shared ancestry of weed progenitor groups, and despite convergence of the trait at the phenotypic level, shattering in U.S. weedy rice may be due to different genetic changes.

\section{The potential for shared loci in divergent weedy traits}

Despite predictions that convergence of weed-adaptive traits should occur among weeds evolving in agricultural settings, we have found divergence for several traits among our closely related weedy rice groups. In particular, SH weeds flower significantly earlier and BHA weeds significantly later than their cultivated progenitors in growth chamber conditions, and $\mathrm{SH}$ weeds tend to be shorter and BHA weeds taller than their ancestors (Reagon et al. 2011) (Table S1, Supporting information). These differences translate into divergence among weed groups also reported in the field (Shivrain et al. 2010). These phenotypic patterns suggest that despite identical environmental conditions, multiple 'adaptive solutions' exist for weedy phenotypes.

Mutations in shared genes could underlie divergent traits if shared signalling and/or metabolic pathways shape alternative trait features (Hodin 2000). Under this scenario, mutations would not be shared among divergent groups, but different mutations in the same gene(s) could underlie divergent phenotypes. Alternatively, similar mutations in similar genes could lead to divergent phenotypes through epistatic interactions with divergent genetic backgrounds. We thus looked for any evidence of shared QTL between the B and S populations for plant height and heading date.

The similar distribution patterns in plant height between populations suggested similar genetic architectures for this trait. However, QTL locations were not shared between mapping populations, and effect directions were not always predictable from parental phenotypes. We identified two QTL in the B population where weedy alleles increase height, yet in the $S$ population, the weedy allele decreased height at only one of three loci. A major locus controlling plant height in cultivated rice has been identified as SD1, within which a large deletion causes a semi-dwarf phenotype employed in breeding during the green revolution (Monna et al. 
2002). We expected to detect this QTL in both populations, as our cultivated parent contains this deletion (Reagon et al. 2011). One of the QTL identified in the B population is physically close to SD1, indicating that it may not be specific to evolution of plant height in the BHA lineage. In contrast, the three QTL in the S population may have contributed to evolution of plant height in the SH lineage. These QTL may come from standing variation in the crop or wild rice, as previously reported height QTL appear to be near these.

Among growth-related traits, phenotypic divergence between weedy groups and between weeds and their cultivated ancestors is most apparent for flowering time (Reagon et al. 2011). Our two mapping populations do not share similar phenotypic distributions for this trait, with the involvement of a single major-effect locus and a few minor-effect loci suggested for the $S$ population, and multiple weaker-effect loci suggested for the B population. Surprisingly, our results indicate that heading date is the trait with most potential for similar genes underlying evolutionary changes in both weed groups. Two of the QTL identified in the $S$ population and two in the B, are located on chromosome 8 , and one set of QTL shares a neighbouring marker across populations. Consistent with the switch to later flowering exhibited by the $\mathrm{SH}$ parent in the greenhouse, in three of the four cases the weedy allele increases days to heading.

Flowering in rice is controlled by several genes that interact to create a wide range in heading dates across different environments (Takahashi et al. 2009). In particular, variations in $H d 1$, which encodes a zinc-finger domain protein (Yano et al. 2000), have been implicated as major regulators of flowering time (Takahashi et al. 2009). A cursory look at $H d 1$ coding region alleles in weedy rice suggests the involvement of Hd1 in weed flowering. Our BHA and cultivated parents have haplotypes containing deletions known to result in nonfunctional haplotypes and photoperiod insensitivity (Takahashi et al. 2009; C. S. Thurber \& A. L. Caicedo, unpublished data). In contrast, our $\mathrm{SH}$ parent has an intact haplotype common in cultivated rice (C. S. Thurber \& A. L. Caicedo, unpublished data), and known to cause photoperiod sensitivity and short day flowering in cultivated rice (Yano et al. 2000; Takahashi et al. 2009). Our mapping results suggest that $H d 1$ does not mediate differences in flowering time between weed parents and between weeds and indica under the variable, primarily long day conditions in our greenhouse. Given that our planting time reflects the timing of planting in Southern U.S. rice fields, our results also suggest that a novel locus or set of loci on chromosome 8 underlie flowering time differences between weed groups in the field and are likely responsible for divergence of weed groups from their cultivated ancestors. These heading dates,
QTL may also be involved in variation in flowering time in cultivated and wild rice, given previously identified QTL and a cloned candidate gene in the region.

\section{The potential for shared loci involved in reversals to wild phenotypes}

Three of our traits show a clear reversal of cultivated phenotypes (non-shattering, straw coloured hulls, no awns) to wild phenotypes (shattering, black hulls, long awns). Due to the diversity of the cultivated ancestral gene pool, as seen by the wide range of hull and awn morphologies in our collection of aus/indica cultivars, it is possible that genes involved in some weedy traits could have arisen from standing ancestral variation. Alternatively, although lack of a role for wild rice sh4 alleles in shattering of weedy rice has been demonstrated, genes underlying hull colour and awn presence in the wild ancestor of rice could be involved in weedy phenotypes through introgression or compensatory mutations that reverse the phenotype in weeds. Thus, weedy convergent genetic evolution can be envisioned at another level: weeds may also share genes underlying weedy traits with wild or cultivated rice.

We checked for evidence of shared genetic changes by examining published QTL from studies involving crosses of wild and cultivated Oryza groups. Seed shattering QTL have been mapped to nearly every rice chromosome, yet only two of our four QTL potentially overlap previously published QTL, both identified in wild by cultivated rice crosses. The potential sharing of some QTL with wild rice suggests that the transition from non-shattering to shattering during weed de-domestication may involve some similar genes as the loss of shattering during domestication.

Although our QTL for awns did not overlap with any other published QTL, our hull colour QTL on chromosome 4 may be the recently cloned Bh4 locus (Zhu et al. 2011). Hull colour in Oryza can vary from light (nearly white and straw) to medium (gold furrowed or brown) to dark (black); this trait may be important for seed dormancy, camouflage and seed dispersal (Zhu et al. 2011). Bh4 encodes an amino acid transporter, and multiple deletions and SNPs seem to be involved in the transition from black hulls in wild rice to straw hulls in cultivated rice (Zhu et al. 2011). Our results suggest that for this weedy trait, causal alleles could be shared with wild rice.

\section{Convergent evolution among global populations of weedy rice}

A few other studies have involved mapping weed adaptive traits in crosses between non-U.S. weedy rice 
and cultivated rice, although with no knowledge of the evolutionary relationship between parents. One such study mapped several traits (e.g. seed shattering, heading date, plant height and yield components) in a weedy rice from France crossed to a japonica cultivar (Bres-Patry et al. 2001), while another set of studies examined seed dormancy, shattering, awns and hull colour in a weedy rice from Thailand crossed to an indica (Gu et al. 2005). We do not share any QTL for overlapping traits with either study, suggesting that convergent genetic evolution may not be the norm among worldwide weedy rice populations.

\section{Caveats and future directions}

Low marker density was apparent in each of our cropweed crosses, consistent with the recent divergence of parental groups (Reagon et al. 2010). Although low marker density decreases statistical power and can lead to difficulties detecting QTL, especially those of small effect, we discovered QTL for most of our weedy traits. Moreover, since divergence time between weeds and their crop ancestors is likely only a few hundred to a few thousand years old (Reagon et al. 2010), it is reasonable to expect a limited number of genes involved in trait divergence among crops and weeds, with most of these having moderate to large effects. Thus, despite the biological constraints leading to low power in our study, and the likelihood that some QTL were not detected, the QTLs discovered here are likely informative about weedy rice evolution.

To determine whether genetic convergence has occurred among weedy groups as a whole, alleles underlying weedy traits must be shown to be shared or present as fixed differences at the population level. Because polymorphism detected between parents may not necessarily represent fixed differences among populations, our study constitutes an initial approach at characterizing the extent of convergent evolution between weed groups. However, the fact that both weed groups have undergone population bottlenecks and have low levels of genetic diversity (Reagon et al. 2010), increases the chances that QTL detected in our crosses may represent true cases of convergent or divergent evolution among populations.

The QTL we detected give us a starting point for identifying genes involved in weed-adaptive traits. A caveat to consider in our study is the lack of a cross between a BHA weed and its putative aus progenitor. Due to the relationship among cultivated and weed groups, QTL detected from the BHA-indica cross could include genomic regions that differ between BHA and aus and regions that differ between indica and aus. Fortunately, this does not hurt our ability to detect QTL relevant to weed evolution, and ongoing attempts to create a BHA-aus cross will help us determine which QTL are specific to BHA weeds.

This study represents a first step towards dissecting the extent of convergent evolution in weed adaptive traits of a potent agricultural weed. Our finding of predominant lack of convergent genetic evolution for shattering, one of the most characteristic traits of weedy rice, joins others in showing that close evolutionary relationships do not imply use of the same genes in adaptation (Arendt \& Reznick 2007). Conversely, shared genetic pathways can be implicated in the evolution of divergent phenotypes, as is likely for flowering time in weeds. Further fine mapping of genes underlying adaptive traits in weedy rice groups, characterization of weed allele frequencies in weedy populations, and search for weed alleles in wild and cultivated ancestors, will contribute to our eventual understanding of the circumstances under which convergent genetic evolution occurs across multiple taxa.

\section{Acknowledgements}

We thank C. Albertson and S. Hazen for help with QTL mapping questions, and S. Perera and R. Dos Santos for help with genotyping and/or phenotyping. This project was funded in part by a grant from the U.S. National Science Foundation Plant Genome Research Program (IOS-1032023) to A.L.C., Kenneth M. Olsen and Y.J.

\section{References}

Arendt J, Reznick D (2007) Convergence and parallelism reconsidered: what have we learned about the genetics of adaptation? Trends in Ecology \& Evolution, 23, 26-32.

Asano K, Takashi T, Miura K et al. (2007) Genetic and Molecular Analysis of Utility of $s d 1$ Alleles in Rice Breeding. Breeding Science, 57, 53-58.

Basu C, Halfhill MD, Mueller TC, Stewart CN Jr (2004) Weed genomics: new tools to understand weed biology. Trends in Plant Science, 9, 391-398.

Bres-Patry C, Lorieuz M, Clement G, Bangratz M, Ghesquiere A (2001) Heredity and genetic mapping of domesticationrelated traits in a temperate japonica weedy rice. Theoretical and Applied Genetics, 102, 118-126.

Cai HW, Morishima H (2000) Genomic regions affecting seed shattering and seed dormancy in rice. Theoretical and Applied Genetics, 100, 840-846.

Cai HW, Morishima H (2002) QTL clusters reflect character associations in wild cultivated rice. Theoretical and Applied Genetics, 104, 1217-1228.

Cai H-Y, Diao S, He Y-G et al. (2012) Genetic and physical mapping of qHY-8, a pleiotropic QTL for heading date and yield-related traits in rice. Euphytica, 184, 109-118.

Caicedo AL, Purugganan MD (2005) Comparative plant genomics. Frontiers and prospects. Plant Physiology, 138, 545-547. 
Caicedo AL, Williamson SH, Hernandez RD et al. (2007) Genome-wide patterns of nucleotide polymorphism in domesticated rice. PLoS Genetics, 3, e163.

Chen X, Temnykh S, Xu Y, Cho YG, McCouch SR (1997) Development of a microsatellite framework map providing genome-wide coverage in rice (Oryza sativa L.). Theoretical and Applied Genetics, 95, 553-567.

Delouche JC, Burgos NR, Gealy DR et al. (2007) Weedy rices- origin, biology, ecology and control (ed. FAO). FAO, Rome, Italy.

Ellstrand NC, Heredia SM, Leak-Garcia JA et al. (2010) Crops gone wild: evolution of weeds and invasives from domesticated ancestors. Evolutionary Applications, 3, 494-504.

Garris AJ, Tai TH, Coburn J, Kresovich S, McCouch S (2005) Genetic structure and diversity in Oryza sativa L. Genetics, 169, 1631-1638.

Gu X-Y, Kianian SF, Hareland GA, Hoffer BL, Foley ME (2005) Genetic analysis of adaptive syndromes interrelated with seed dormancy in weedy rice (Oryza sativa). Theoretical and Applied Genetics, 110, 1108-1118.

Harlan JR, DeWet JM (1965) Some thoughts about weeds. Economic Botany, 19, 16-24.

Hodin J (2000) Plasticity and constraints in development and evolution. Journal of Experimental Zoology, 288, 1-20.

Lee S-J, Oh C-S, Suh J-P, McCouch SR, Ahn S-N (2005) Identification of QTLs for domestication-related and agronomic traits in an Oryza sativa $\times$ O. rufipogon BC1F7 population. Plant Breeding, 124, 209-219.

Li C, Zhou A, Sang T (2006a) Genetic analysis of rice domestication syndrome with the wild annual species, Oryza nivara. New Phytologist, 170, 185-194.

Li C, Zhou A, Sang T (2006b) Rice domestication by reducing shattering. Science, 311, 1936-1939.

Li S-B, Zhang Z-H, Hu Y et al. (2006c) Genetic dissection of developmental behavior of crop growth rate and its relationships with yield and yield related traits in rice. Plant Science, 170, 911-917.

Lin Z, Griffith ME, Li X et al. (2007) Origin of seed shattering in rice (Oryza sativa L.). Planta, 226, 11-20.

Londo JP, Schaal BA (2007) Origins and population genetics of weedy red rice in the USA. Molecular Ecology, 16, 45234535.

Manceau M, Domingues VS, Linnen CR, Rosenblum EB, Hoekstra HE (2010) Convergence in pigmentation at multiple levels: mutations, genes and function. Philosophical Transactions of the Royal Society of London. Series B, Biological Sciences, 365, 2439-2450.

Manichaikul A, Palmer AA, Sen S, Broman KW (2007) Significance threshold for quantitative trait locus mapping under selective genotyping. Genetics, 177, 1963-1966.

McCouch SR, Teytelman L, Xu Y et al. (2002) Development and mapping of 2240 new SSR markers for rice (Oryza sativa L.). DNA Research, 9, 199-207.

Monna L, Kitazawa N, Yoshino R et al. (2002) Positional cloning of rice semidwarfing gene, sd-1: rice "green revolution gene" encodes a mutant enzyme involved in gibberellin synthesis. DNA Research, 9, 11-17.

Noldin JA, Chandler JM, McCauley GN (1999) Red rice (Oryza sativa) biology. I. Characterization of red rice ecotypes. Weed Technology, 13, 12-18.
Olsen KM, Caicedo AL, Jia Y (2007) Evolutionary genomics of weedy rice in the USA. Journal of Integrative Plant Biology, 49, 811-816.

Panaud O, Chen X, McCouch SR (1996) Development of microsatellite markers and characterization of simple sequence length polymorphism (SSLP) in rice (Oryza sativa L.). Molecular and General Genetics, 252, 597-607.

Powles SB, Yu Q (2010) Evolution in action: plants resistant to herbicides. Annual Review of Plant Biology, 61, 317-347.

Purugganan MD, Fuller DQ (2009) The nature of selection during plant domestication. Nature, 457, 843-848.

Reagon M, Thurber CS, Gross BL et al. (2010) Genomic patterns of nucleotide diversity in parallel populations of U.S. weedy rice. BMC Evolutionary Biology, 10, 180.

Reagon M, Thurber CS, Olsen KM, Jia Y, Caicedo AL (2011) The long and the short of it: SD1 polymorphism and the evolution of growth trait divergence in U.S. weedy rice. Molecular Ecology, 20, 3743-3756.

Remigereau M-S, Lakis G, Rekima S et al. (2011) Cereal domestication and evolution of branching: evidence for soft selection in the Tb1 orthologue of Pearl Millet (Pennisetum glaucum [L.] R. Br.). PLoS ONE, 6, e22404.

Rompler H, Rohland N, Lalueza-Fox C et al. (2006) Nuclear gene indicates coat-color polymorphism in Mammoths. Science, 313, 62.

Schluter D, Clifford EA, Nemethy M, McKinnon JS (2004) Parallel evolution and inheritance of quantitative traits. American Naturalist, 163, 809-822.

Septiningsih EM, Prasetiyono J, Lubis E et al. (2003) Identification of quantitative trait loci for yield and yield components in an advanced backcross population derived from the Oryza sativa variety IR64 and the wild relative O. rufipogon. Theoretical and Applied Genetics, 107, 1419-1432.

Shen Y-J, Jiang H, Jin J-P et al. (2004) Development of genomewide DNA polymorphism database for map-based cloning of rice genes. Plant Physiology, 135, 1198-1205.

Shivrain VK, Burgos NR, Gealy DR, Sales MA, Smith KL (2009) Gene flow from weedy red rice (Oryza sativa L.) to cultivated rice and fitness of hybrids. Pest Management Science, 65, 1124-1129.

Shivrain VK, Burgos NR, Agrama HA et al. (2010) Genetic diversity of weedy red rice (Oryza sativa) in Arkansas, USA. Weed Research, 50, 289-302.

Smith SD, Rausher MD (2011) Gene loss and parallel evolution contribute to species difference in flower color. Molecular Biology and Evolution, 28, 2799-2810.

Steiner CC, Rompler H, Boettger LM, Shoneberg T, Hoekstra HE (2008) The genetic basis of phenotypic convergence in beach mice: similar pigment patterns but different genes. Molecular Biology and Evolution, 26, 35-45.

Stinchcombe JR, Hoekstra HE (2008) Combining population genomics and quantitative genetics: finding the genes underlying ecologically important traits. Heredity, 100, 158170 .

Takahashi Y, Teshima KM, Yokoi S, Innan H, Shimamoto K (2009) Variations in Hd1 proteins, Hd3a promoters, and Ehd1 expression levels contribute to diversity of flowering time in cultivated rice. Proceedings of the National Academy Sciences, USA, 106, 4555-4560. 
Tan WD, Gan FF, Chang TC (2004) Using normal quantile plot to select an appropriate transformation to achieve normality. Computational Statistics and Data Analysis, 45, 609-619.

Thomson MJ, Tai TH, McClung AM et al. (2003) Mapping quantitative trait loci for yield, yield components and morphological traits in an advanced backcross population between Oryza rufipogon and the Oryza sativa cultivar Jefferson. Theoretical and Applied Genetics, 107, 479-493.

Thurber CS, Reagon M, Gross BL et al. (2010) Molecular evolution of shattering loci in U.S. weedy rice. Molecular Ecology, 19, 3271-3284.

Thurber CS, Hepler P, Caicedo AL (2011) Timing is everything: early degradation of abscission layer may lead to increased shattering in U.S. weedy rice. BMC Plant Biology, 11, 14.

Tilquin P, Coppieters W, Elsen JM et al. (2001) Statistical power of QTL mapping methods applied to bacteria counts. Genetical Research, 78, 303-316.

Vaughan LK, Ottis BV, Prazak-Havey AM et al. (2001) Is all red rice found in commercial rice really Oryza sativa? Weed Science, 49, 468-476.

Wang S, Basten CJ, Zeng Z-B (2012) Windows QTL Cartographer 2.5. Department of Statistics, North Carolina State University, Raleigh, NC. (http://statgen.ncsu.edu/qtlcart/WQTL Cart.htm)

Wei X, Xu J, Guo H et al. (2010) DTH8 suppresses flowering in rice, influencing plant height and yield potential simultaneously. Plant Physiology, 153, 1747-1758.

Xiao JH, Li J, Grandillo S et al. (1998) Identification of traitimproving quantitative trait loci alleles from a wild rice relative, Oryza rufipogon. Genetics, 150, 899-909.

Xu NW, Xu S, Ehlers J (2009) Estimating the broad-sense heritability of early growth cowpea. International Journal of Plant Genomics, doi:10.1155/2009/984521.

Yan W-H, Wang P, Chen H-X et al. (2011) A major QTL, Ghd8, plays pleiotropic roles in regulating grain productivity, plant height, and heading date in rice. Molecular Plant, 4, 319-330.

Yano M, Harushima Y, Nagamura Y et al. (1997) Identification of quantitative trait loci controlling heading date in rice using a high-density linkage map. Theoretical and Applied Genetics, 95, 1025-1032.

Yano M, Katayose Y, Ashikari M et al. (2000) Hd1, a major photoperiod sensitivity quantitative trait locus in rice, is closely related to the Arabidopsis flowering time gene CONSTANS. The Plant Cell, 12, 2473-2483.

Yoon H-S, Baum DA (2004) Transgenic study of parallelism in plant morphological evolution. Proceedings of the National Academy of Sciences, USA, 101, 6524-6529.

Zhang L-B, Zhu Q, Wu Z-Q et al. (2009) Selection on grain shattering genes and rates of rice domestication. New Phytologist, 184, 708-720.

Zhang L, Wang S, Li H et al. (2010) Effects of missing marker and segregation distortion on QTL mapping in F2 populations. Theoretical and Applied Genetics, 121, 1071-1082.
Zhu B-F, Si L, Wang Z et al. (2011) Genetic control of a transition from black to straw-white seed hull in rice domestication. Plant Physiology, 155, 1301-1311.

Zhu Y, Ellstrand NC, Lu B-R (2012) Sequence polymorphism in wild, weedy, and cultivated rice suggest seed-shattering locus sh4 played a minor role in Asian rice domestication. Ecology and Evolution, 2, 2106-2113.

A.L.C. and C.S.T. designed the research. C.S.T. performed the research. M.H.J. and Y.J. contributed reagents and data collection. A.L.C. and C.S.T. analysed the data. A.L.C. and C.S.T wrote the article.

\section{Data accessibility}

Phenotype and genotype data for all individuals are included in the Supplemental Data files.

\section{Supporting information}

Additional supporting information may be found in the online version of this article.

Fig. S1 LOD plots for quantitative traits (seed shattering, heading date, plant height) across all twelve chromosomes in both mapping populations.

Fig. S2 LOD plots for the qualitative traits (hull colour, awns) across all twelve chromosomes in the $\mathrm{B}$ mapping population.

Table S1 Means and standard deviations (in parenthesis) of phenotypes measured in cultivated and weedy populations.

Table S2 Phenotype values for the S population individuals.

Table S3 Phenotype values for the B population individuals.

Table S4 Pearson's correlations between traits in the $F_{2}$ populations. Only significant correlations are shown. Bold represents correlations significant at $P<0.01$, all others are significant at $P<0.05$.

Table S5 Broad-sense heritability values for the quantitative traits studied.

Table S6 Genotypes of the B population individuals at all markers used for mapping.

Table S7 Genotypes of the S population individuals at all markers used for mapping. 
Hull color

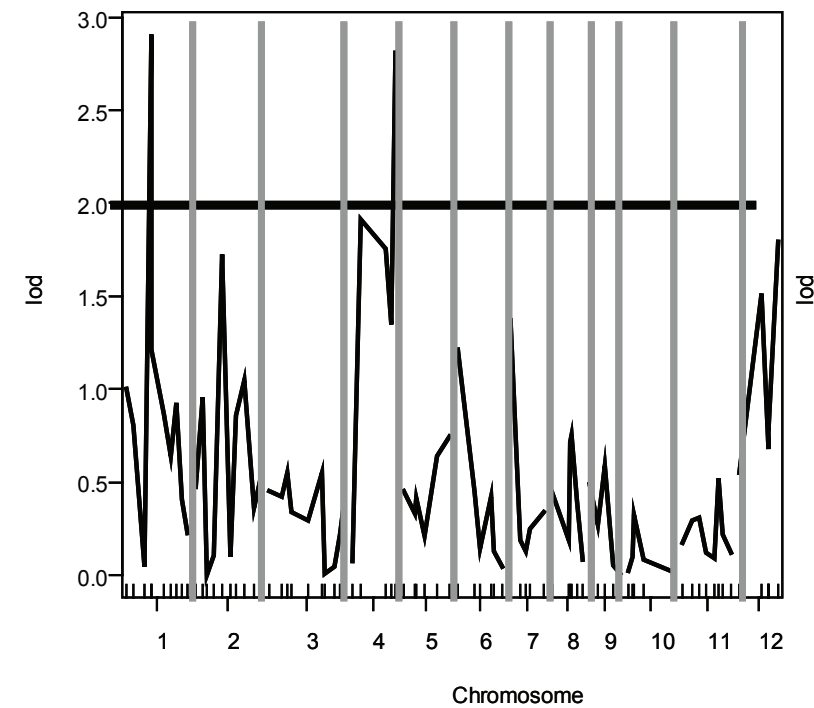

Awns

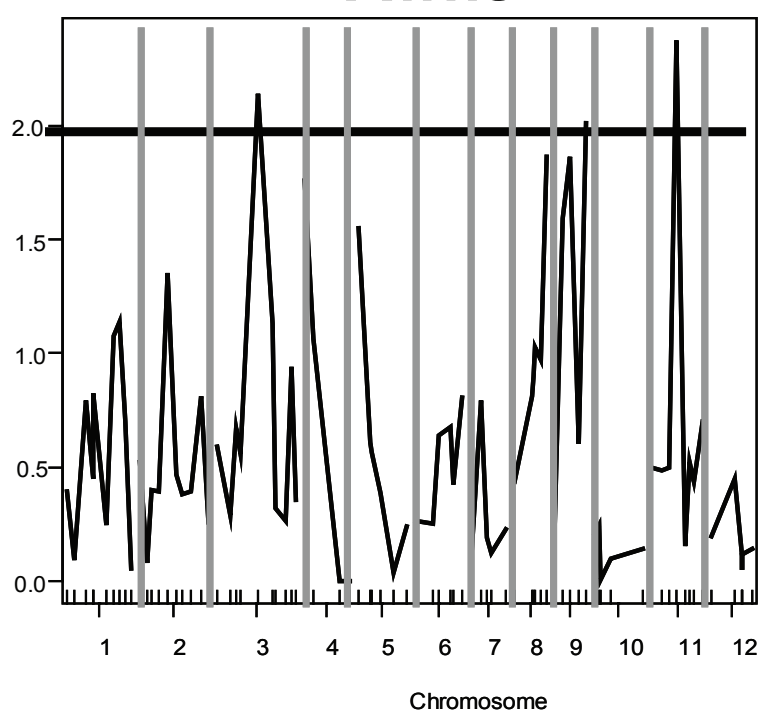

